\title{
Identification of therapeutic targets for Alzheimer's disease via differentially expressed gene and weighted gene co-expression network analyses
}

\author{
YUJIE JIA $^{1}$, KUN NIE $^{1}$, JING LI $^{1}$, XINYUE LIANG ${ }^{2}$ and XUEZHU ZHANG ${ }^{1}$ \\ ${ }^{1}$ Institute of Acupuncture and Moxibustion, First Teaching Hospital of Tianjin University of Traditional Chinese Medicine; \\ ${ }^{2}$ Personnel Department, Tianjin University of Traditional Chinese Medicine, Tianjin 300193, P.R. China
}

Received September 22, 2015; Accepted October 4, 2016

DOI: $10.3892 / \mathrm{mmr} .2016 .5828$

\begin{abstract}
In order to investigate the pathogenic targets and associated biological process of Alzheimer's disease in the present study, mRNA expression profiles (GSE28146) and microRNA (miRNA) expression profiles (GSE16759) were downloaded from the Gene Expression Omnibus database. In GSE28146, eight control samples, and Alzheimer's disease samples comprising seven incipient, eight moderate, seven severe Alzheimer's disease samples, were included. The Affy package in $\mathrm{R}$ was used for background correction and normalization of the raw microarray data. The differentially expressed genes (DEGs) and differentially expressed miRNAs were identified using the Limma package. In addition, mRNAs were clustered using weighted gene correlation network analysis, and modules found to be significantly associated with the stages of Alzheimer's disease were screened out. The Database for Annotation, Visualization, and Integrated Discovery was used to perform Gene Ontology and Kyoto Encyclopedia of Genes and Genomes pathway analyses. The target genes of the differentially expressed miRNAs were identified using the miRWalk database. Compared with the control samples, 175,59 genes and 90 DEGs were identified in the incipient, moderate and severe Alzheimer's disease samples, respectively. A module, which contained 1,592 genes was found to be closely associated with the stage of Alzheimer's disease and biological processes. In addition, pathways associated with Alzheimer's disease and other neurological diseases were found to be enriched in those genes. A total of 139 overlapped genes were identified between those genes and the DEGs in the three groups. From the miRNA expression profiles, 189 miRNAs were found
\end{abstract}

Correspondence to: Professor Xuezhu Zhang, Institute of Acupuncture and Moxibustion, First Teaching Hospital of Tianjin University of Traditional Chinese Medicine, 314 Anshanxi Road, Nankai, Tianjin 300193, P.R. China

E-mail: xuezhuzhang1999@sina.com

Key words: Alzheimer's disease, Gene Expression Omnibus, pathogenic targets, neurological diseases differentially expressed in the samples from patients with Alzheimer's disease and 1,647 target genes were obtained. In addition, five overlapped genes were identified between those 1,647 target genes and the 139 genes, and these genes may be important pathogenic targets for Alzheimer's disease. Through bioinformatics analysis, potential therapeutic targets and associated biological processes were identified, which may assist in understanding and treatment of Alzheimer's disease.

\section{Introduction}

The preservation of cognitive function during aging is becoming an increasingly critical public health problem and emerging as one of the major medical challenges of the 21st century, particularly in countries with rapidly aging populations $(1,2)$. Alzheimer's disease, the most frequent cause of dementia, is an age-related neurodegenerative disease, which is characterized by amyloid- $\beta(\mathrm{A} \beta)$ peptide deposition extracellularly and hyperphosphorylated tau protein accumulation intracellularly as neurofibrillary tangles $(3,4)$. Although $1-2 \%$ of cases of Alzheimer's disease are derived from pathogenic mutations in amyloid precursor protein (APP) and presenilin $1 / 2$ genes, the majority of cases are sporadic and tend to result from pathogenic interactions between multiple factors, including APP/A $\beta$ aging, genetic variants and apolipoprotein E genotype (5). Although these previous investigations indicate notable links between certain genes and Alzheimer's disease, the exact mechanism underlying the progression of Alzheimer's disease remains to be fully elucidated.

Genome-wide approaches have reshaped the landscape of medical investigations. One of the key mechanisms by which gene expression is controlled, is examined via a complicated process, termed transcriptional regulation, which is mediated through transcription factors. However, the identification of a class of small non-coding RNAs, termed microRNAs (miRNAs), has augmented the complexity of transcriptional regulation and opened a novel field of medical investigations. miRNAs, which consist of 18-24 nucleotides, frequently bind to the 3'-untranslated region (3'-UTR) of target transcripts and regulate gene expression by degrading the target mRNA or inhibiting its translation. They are associated with several biological processes, including development, apoptosis and 
proliferation, and their differential expression has been detected in several disorders, including different types of cancer (6), stroke (7), diabetes (8), and neurological (9), and autoimmune diseases $(10,11)$. Compared with differential expression analysis, network analysis may offer an improved method of revealing the correlation between genes and phenotypes of interest, which are increasingly used in systems biology. Weighted gene co-expression network analysis (WGCNA), which was developed by Langfelder and Horvath (12), describes the correlations among genes and forms gene modules according to their expression values across microarray samples. However, reports on the association between miRNAs and Alzheimer's disease using a high throughput method are limited. For this reason, it is important to identify and analyze miRNAs, which are likely to be significant in the expression of disease-associated genes. The aim of the present study was to identify key genes and biological processes potentially associated with the progression of Alzheimer's disease, which may aid development of small, bioactive molecules. The present study provides important data and theoretical support for investigations of the physiological and pathological processes of Alzheimer's disease.

\section{Materials and methods}

Microarray dataset. The mRNA expression profile of GSE28146 (13) and the miRNA expression profile of GSE16759 (14) were downloaded from the Gene Expression Omnibus (GEO) database (http://www.ncbi.nlm.nih. gov/geo/). In GSE28146, eight control samples, and samples of Alzheimer's disease comprising seven incipient, eight moderate and seven severe samples were included. Total RNA was extracted from the formalin-fixed-paraffin-emb edded hippocampus (from the GEO public database), and hybridized in the GPL570 Affymetrix HGU 133 Plus 2 microarray (Affymetrix, Inc., Santa Clara, CA, USA). For GSE16759, the miRNA and mRNA expression were profiled for four control samples and four Alzheimer's disease samples. In the present study, only those miRNA expression profiles detected via the GPL8757 USC/XJZ Human 0.9 K miRNA-940-v1.0 microarray platform (www.chibi. ubc.ca/Gemma/arrays/showArrayDesign.html?id=680) were used in the subsequent analysis.

Microarray data analysis. The raw data were converted into a recognizable format in $\mathrm{R}$ software. The Affy (15) package (bioconductor.org/packages/release/bioc/html/affy.html) was used for the background correction and normalization of the data. The differentially expressed mRNAs and miRNAs (DEmiRNAs) between the Alzheimer's disease and control samples were identified using the Limma (16) package (bioconductor.org/packages/release/bioc/html/limma.html) with the following criteria: False discovery rate $<0.05$ and $\mid \log 2$ (fold change) $\mid>1$.

Weighted gene co-expression network analysis (WGCNA) of the mRNA microarray. The WGCNA package of $\mathrm{R}$ was used for clustering of the first 5,000 genes with the highest coefficient of variation [CV; (standard deviation) / (mean
Table I. Numbers of total, upregulated and downregulated DEGs in the three Alzheimer's disease sample groups.

\begin{tabular}{lccc}
\hline $\begin{array}{l}\text { Sample } \\
\text { group }\end{array}$ & $\begin{array}{c}\text { Total } \\
\text { DEGs (n) }\end{array}$ & Upregulated & Downregulated \\
\hline Incipient & 175 & 53 & 122 \\
Moderate & 59 & 22 & 37 \\
Severe & 90 & 31 & 59 \\
\hline
\end{tabular}

DEGs, differentially expressed genes.

value) $x 100 \%$ )] between Alzheimer's disease in the mRNA microarray. Age, sex and disease stage were screened out based on the correlation coefficient and P-value, which were considered to be the clinical data and gene modules closely associated with Alzheimer's disease. Gene Ontology (GO; geneontology.org) and Kyoto Encyclopedia of Genes and Genomes (KEGG; www.genome.jp/kegg) pathway enrichment analysis were performed using the Database for Annotation, Visualization and Integrated Analysis (DAVID; (http://david.abcc.ncifcrf.gov/) in the hub module (most significant module in the network).

Screening target genes of DEmiRNAs. The validated target genes of the DEmiRNAs were obtained using the miRWalk database (zmf.umm.uni-heidelberg.de/apps/zmf/mirwalk2) based on the combination of miRanda, TargetScan, Pictar and miRbase (17). In addition, the overlap among target genes, genes in the hub module and the DEGs were identified. The miRNA-mRNA regulation network was constructed based on the overlapped genes and their upstream miRNAs, and visualized using Cytoscape software version 2.6.3 (www. cytoscape.org) (18).

\section{Results}

DEGs and DEmiRNAs. A total of 17,559 genes and 90 DEGs were obtained in the incipient, moderate and severe Alzheimer's disease samples, respectively, compared with control samples. The numbers of upregulated and downregulated genes are listed in Table I. In addition, 164 DEmiRNAs, which contained 74 downregulated and 90 upregulated DEmiRNAs were identified in the Alzheimer's disease samples.

Hub modules of Alzheimer's disease. Through the WGCNA, the first 5,000 genes with the highest $\mathrm{CV}$ between the control samples and Alzheimer's disease samples in the mRNA microarray were divided into five gene modules. The largest of these modules contained 2,200 genes, whereas only 52 genes were included in the smallest module. A total of 787 genes were not included in any of the five modules. The correlation coefficients between every gene module, and the age, sex and stage of Alzheimer's disease were also obtained. The distribution of genes in the five gene modules and the importance of each gene module are shown in Fig. 1A and $\mathrm{B}$, respectively. 
Table II. Top 10 significant GO terms enriched in genes of the blue module.

\begin{tabular}{llrr}
\hline Category & \multicolumn{1}{c}{ GO term } & Number of Genes & P-value \\
\hline BP & Transmission of nerve impulse & 64 & $7.00 \times 10^{-11}$ \\
BP & Synaptic transmission & 55 & $1.28 \times 10^{-9}$ \\
CC & Mitochondrion & 61 & $4.21 \times 10^{-9}$ \\
CC & Synapse & 24 & $6.76 \times 10^{-9}$ \\
CC & Synaptic vesicle & 87 & $7.41 \times 10^{-9}$ \\
CC & Mitochondrial part & 59 & $1.02 \times 10^{-9}$ \\
CC & Neuron projection & 137 & $1.06 \times 10^{-9}$ \\
CC & Organelle membrane & 47 & $1.34 \times 10^{-9}$ \\
CC & Synapse part & 52 & $1.73 \times 10^{-8}$ \\
BP & Generation of precursor metabolites and energy &
\end{tabular}

GO, Gene Ontology; BP, biological process; CC, cellular component.

$\mathbf{A}$

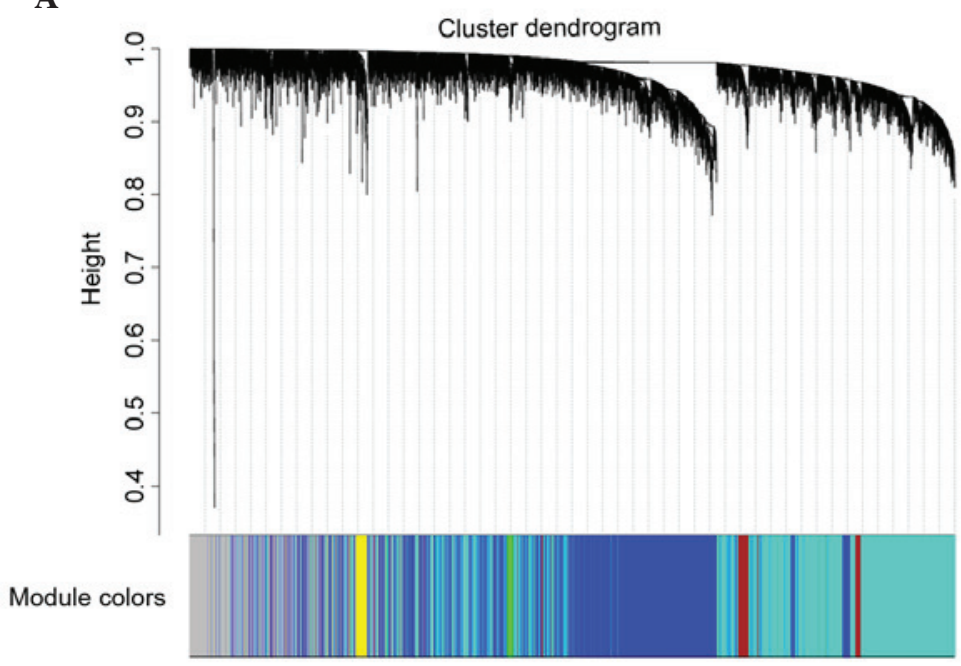

B

\begin{tabular}{|c|c|c|c|c|}
\hline \multirow[b]{2}{*}{ MEturquoise } & \multicolumn{3}{|c|}{ Module-trait relationships } & \multirow[b]{2}{*}{$\pi^{1}$} \\
\hline & $\begin{array}{c}-0.028 \\
(0.9)\end{array}$ & $\begin{array}{l}-0.19 \\
(0.3)\end{array}$ & $\begin{array}{l}0.14 \\
(0.5)\end{array}$ & \\
\hline MEyellow & $\begin{array}{c}0.0091 \\
\text { (1) }\end{array}$ & $\begin{array}{c}-0.48 \\
(0.007)\end{array}$ & $\begin{array}{c}0.029 \\
(0.9)\end{array}$ & -0.5 \\
\hline MEblue & $\begin{array}{l}-0.11 \\
(0.6)\end{array}$ & $\begin{array}{l}0.19 \\
(0.3)\end{array}$ & $\begin{array}{l}-0.46 \\
(0.01)\end{array}$ & \\
\hline MEbrown & $\begin{array}{c}-0.097 \\
(0.6)\end{array}$ & $\begin{array}{l}0.15 \\
(0.4)\end{array}$ & $\begin{array}{c}-0.13 \\
(0.5)\end{array}$ & \\
\hline MEgreen & $\begin{array}{l}0.059 \\
(0.8)\end{array}$ & $\begin{array}{c}0.49 \\
(0.006)\end{array}$ & $\begin{array}{c}-0.021 \\
(0.9)\end{array}$ & -0.5 \\
\hline MEgrey & $\begin{array}{c}-0.33 \\
(0.08)\end{array}$ & $\begin{array}{l}0.13 \\
(0.5)\end{array}$ & $\begin{array}{l}0.27 \\
(0.1)\end{array}$ & -1 \\
\hline & $\mathrm{set}^{t}$ & $p^{8}$ & & \\
\hline
\end{tabular}

Figure 1. Weighted gene co-expression network analysis of the mRNA microarray. (A) Distribution of the 5,000 genes in the five modules. Different colors represent different modules. Gray indicates genes, which were not included in any module. (B) Correlation coefficients (significance shown within brackets), between each module and the phenotypes (sex, age and status of Alzheimer's disease).

As shown in Fig. 1B, the blue module, which contained 1,592 genes, was the most significant module (correlation coefficient $=-0.46 ; \mathrm{P}=0.01$ ) associated with the stage of Alzheimer's disease. Functional enrichment analysis indicated that the GO terms and KEGG pathways associated with the biological processes or diseases of the nervous system were enriched in the genes of the blue module. The top 10 significant GO terms are shown in Table II. Table III shows the 14 enriched KEGG pathways.

Target genes of DEmiRNAs. In the miRWalk database, 746 non-redundant validated target genes were identified for the 164 DEmiRNAs. Five overlaps were obtained, which were regulated by four miRNAs: hsa-mir-376a-2, hsa-mir-376a-1, hsa-mir-15a and hsa-mir-424. The regulation network between those overlapped genes and their upstream miRNAs is shown in Fig. 2.

\section{Discussion}

In the present study, microarray technology was used to identify the therapeutic targets and the associated biological processes for Alzheimer's disease. Using the combination of differentially expressed analysis and WGCNA, several potential targets, including miR-15a and peptidyl-glycine $\alpha$-amidating monooxygenase (PAM) were obtained. In addition, several biological process and KEGG pathways, which were closely associated with Alzheimer's disease, were further validated.

Sprouty (Spry) proteins contain a carboxyl-terminal cysteine-rich domain, which is essential for the inhibitory activity on receptor tyrosine kinase signaling proteins, and they require growth factors to stimulate translocation of the protein to membrane ruffles. Over previous years, members of the Spry family have emerged as critical modulators of 
Table III. Enriched Kyoto Encyclopedia of Genes and Genomes pathways in genes of the blue module.

\begin{tabular}{lcc}
\hline Pathway name & Number of genes & P-value \\
\hline Oxidative phosphorylation & 26 & $2.24 \times 10^{-5}$ \\
Parkinson's disease & 23 & $3.82 \times 10^{-4}$ \\
Huntington's disease & 28 & $8.26 \times 10^{-4}$ \\
Epithelial cell signaling in Helicobacter pylori infection & 13 & $6.43 \times 10^{-3}$ \\
Glycolysis/Gluconeogenesis & 12 & $6.66 \times 10^{-3}$ \\
Alzheimer's disease & 23 & $8.93 \times 10^{-3}$ \\
Vibrio cholerae infection & 11 & 0.0114 \\
Oocyte meiosis & 17 & 0.0123 \\
Amyotrophic lateral sclerosis & 10 & 0.0219 \\
Wnt signaling pathway & 20 & 0.0285 \\
Pathogenic Escherichia coli infection & 10 & 0.0336 \\
Pyruvate metabolism & 8 & 0.0354 \\
Long-term potentiation & 11 & 0.0401 \\
Ubiquitin mediated proteolysis & 18 & 0.0415
\end{tabular}

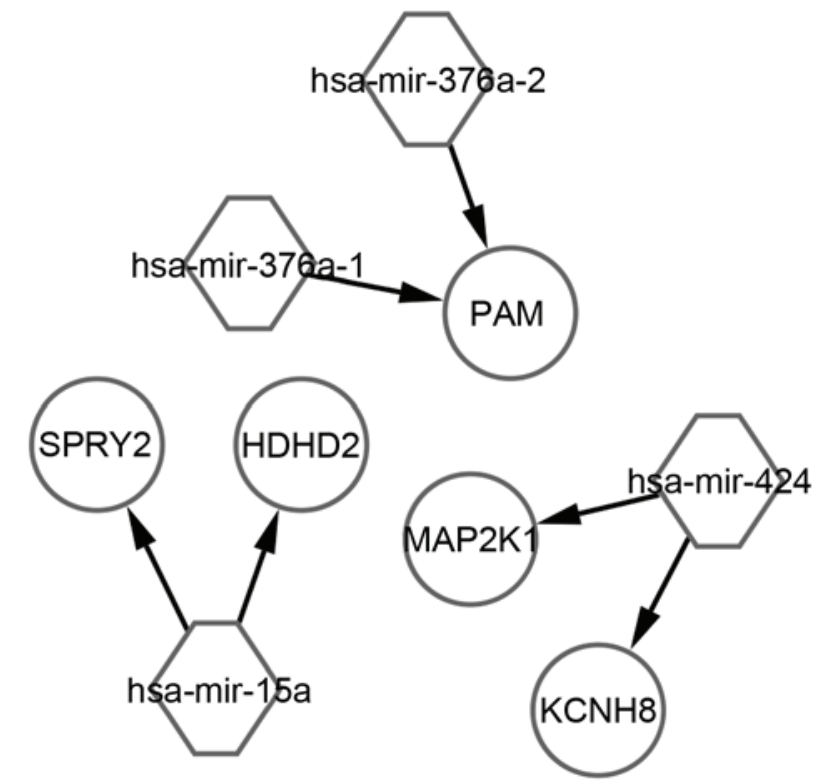

Figure 2. miRNA-mRNA regulation network. Circles represent overlapping genes between target genes, differentially expressed genes and genes in the blue module. Hexagons represent the upstream miRNAs of those overlaps. The arrows represent regulation of miRNAs to genes. miR, microRNA; SPRY2, sprouty 2; HDHD2, haloacid dehalogenase-like hydrolase domain-containing 2; PAM, peptidyl-glycine $\alpha$-amidating monooxygenase; MAP2K1, mitogen-activated protein kinase kinase 1; KCNH8, potassium voltage-gated channel subfamily $\mathrm{H}$, member 8 .

growth factor signaling $(19,20)$. Mammalian spry genes are expressed in restricted patterns in several adult tissues and in the embryo during development. They are important in the development of various organs, including the kidney, lung, testis and tooth (21-25). At the cellular level, the overexpression of Spry proteins antagonizes proliferation, migration and differentiation in response to serum or growth factors in various cell lines $(19,20)$. In addition, Gross et al (26) demonstrated that the overexpression of wild-type Spry2 inhibits neurite formation and expression of neurofilament light chain, whereas the inhibition of Spry2 accelerates sprouting of multiple neurites. These findings suggest that Spry2 is potentially involved in neurological disease. The miR-15a/miR16-1 cluster, located on $13 \mathrm{q}$, is important in regulating cell proliferation, apoptosis and expression in the brain, heart, liver, spleen, lung, kidney, small intestine and testis under physiological conditions (27). Gao et al (28) found that miR-15a is important in the regulation of neuronal maturation, and further confirmed that the overexpression of miR-15a inhibits dendritic morphogenesis in immature neurons, whereas, a reduction in $\mathrm{miR}-15 \mathrm{a}$ has the opposite effect. Another study provided evidence for a potential causal association between the expression of miR-29a/b-1 and generation of $A \beta$, with the loss of specific miRNAs contributing to increased levels of $\beta$-secretase 1 (BACE1) and $A \beta$ in sporadic Alzheimer's disease. Another three miRNAs were also predicted, which had candidate binding sites within the 3'UTRs of BACE1, including miR-15a (also found in the present study), miR-9 and miR-19b (29). In the present study, an important subgroup of patients with Alzheimer's disease exhibited increased protein expression of Spry2 in the tissue of the hippocampus, and Spry2 was predicted as an important disease marker for Alzheimer's disease. The present study also used the prediction algorithms, miRanda, Targetscan, Pictar and miRbase, which confirmed that miR-15a had candidate binding sites within the 3'UTRs of Spry2, which indicated miR-15a as a potential major suppressor of the protein expression of Spry2.

The results of the present study also suggested that miRNA expression profiles were altered in Alzheimer's disease. Whether these changes were specific for Alzheimer's disease, or whether they were a cause or a consequence of the disease process, and whether these data may be used for diagnosis, remain to be investigated. Another two miRNAs, miR-376-a1 and miR-376-a2, were identified as significantly altered, which had candidate binding sites within the 3'UTRs of PAM, whereas one candidate miRNA-binding site, miR-424, was found within the 3' UTRs of mitogen-activated 
protein kinase kinase 1 and potassium voltage-gated channel subfamily $\mathrm{H}$, member 8 .

In conclusion, the findings of the present study suggested a molecular link between miRNA and gene expression potentially involved in Alzheimer's disease. The functions of these genes in Alzheimer's disease require further validation through the use of molecular biology experiments. The present study revealed potential therapeutic targets and associated biological processes through the use of differentially expressed gene analysis and WGCNA, and these findings may assist in the treatment of Alzheimer's disease.

\section{Acknowledgements}

This study was supported by the National Natural Science Foundation of China (grant nos. 81202739, 81473766 and $81173337)$ and the Key Project of Tianjin Natural Science Fund (grant no. 16JCZDJC36200).

\section{References}

1. Sosa AL, Albanese E, Stephan BC, Dewey M, Acosta D, Ferri CP, Guerra M, Huang Y, Jacob KS, Jiménez-Velázquez IZ, et al: Prevalence, distribution, and impact of mild cognitive impairment in Latin America, China and India: A 10/66 population-based study. PLoS Med 9: e1001170, 2012.

2. Ashby-Mitchell K, Jagger C, Fouweather T and Anstey KJ: Life expectancy with and without cognitive impairment in seven Latin American and Caribbean countries. PloS One 10: e0121867, 2015.

3. Price DL, Tanzi RE, Borchelt DR and Sisodia SS: Alzheimer's disease: Genetic studies and transgenic models. Annu Rev Genet 32: 461-493, 1998.

4. Barnes DE and Yaffe K: The projected effect of risk factor reduction on Alzheimer's disease prevalence. Lancet Neurol 10: 819-828, 2011.

5. Huang Y and Mucke L: Alzheimer mechanisms and therapeutic strategies. Cell 148: 1204-1222, 2012.

6. Gregory RI and Shiekhattar R: MicroRNA biogenesis and cancer. Cancer Res 65: 3509-3512, 2005.

7. Tan JR, Tan KS, Koo YX, Yong FL, Wang CW, Armugam A and Jeyaseelan K: Blood microRNAs in low or no risk ischemic stroke patients. Int J Mol Sci 14: 2072-2084, 2013.

8. Balasubramanyam M, Aravind S, Gokulakrishnan K, Prabu P, Sathishkumar C, Ranjani H and Mohan V: Impaired miR-146a expression links subclinical inflammation and insulin resistance in Type 2 diabetes. Mol Cell Biochem 351: 197-205, 2011.

9. Minones-Moyano E, Porta S, Escaramis G, Rabionet R, Iraola S, Kagerbauer B, Espinosa-Parrilla Y, Ferrer I, Estivill X and Martí E: MicroRNA profiling of parkinson's disease brains identifies early downregulation of $\mathrm{miR}-34 \mathrm{~b} / \mathrm{c}$ which modulate mitochondrial function. Hum Mol Genet 20: 3067-3078, 2011.

10. Argyropoulos C, Wang K, McClarty S, Huang D, Bernardo J, Ellis D, Orchard T, Galas D and Johnson J: Urinary microRNA profiling in the nephropathy of type 1 diabetes. PLoS One 8: e54662, 2013.

11. Salas-Pérez F, Codner E, Valencia E, Pizarro C, Carrasco E and Pérez-Bravo F: MicroRNAs miR-21a and miR-93 are down regulated in peripheral blood mononuclear cells (PBMCs) from patients with type 1 diabetes. Immunobiology 218: 733-737, 2013.
12. Langfelder $\mathrm{P}$ and Horvath S: WGCNA: An R package for weighted correlation network analysis. BMC Bioinformatics 9: 559, 2008

13. Blalock EM, Buechel HM, Popovic J, Geddes JW and Landfield PW: Microarray analyses of laser-captured hippocampus reveal distinct gray and white matter signatures associated with incipient Alzheimer's disease. J Chem Neuroanat 42: 118-126, 2011.

14. Nunez-Iglesias J, Liu CC, Morgan TE, Finch CE and Zhou XJ: Joint genome-wide profiling of miRNA and mRNA expression in Alzheimer's disease cortex reveals altered miRNA regulation. PLoS One 5: e8898, 2010.

15. Gautier L, Cope L, Bolstad BM and Irizarry RA: Affy-analysis of affymetrix genechip data at the probe level. Bioinformatics 20: 307-315, 2004

16. Ritchie ME, Phipson B, Wu D, Hu Y, Law CW, Shi W and Smyth GK: Limma powers differential expression analyses for RNA-sequencing and microarray studies. Nucleic Acids Res 43: e47, 2015.

17. Dweep H, Sticht C, Pandey P and Gretz N: miRWalk-database: Prediction of possible miRNA binding sites by 'walking' the genes of three genomes. J Biomed Inform 44: 839-847, 2011.

18. Shannon P, Markiel A, Ozier O, Baliga NS, Wang JT, Ramage D, Amin N, Schwikowski B and Ideker T: Cytoscape: A software environment for integrated models of biomolecular interaction networks. Genome Res 13: 2498-2504, 2003.

19. Mason JM, Morrison DJ, Basson MA and Licht JD: Sprouty proteins: Multifaceted negative-feedback regulators of receptor tyrosine kinase signaling. Trends Cell Biol 16: 45-54, 2006.

20. Kim HJ and Bar-Sagi D: Modulation of signalling by Sprouty: A developing story. Nat Rev Mol Cell Biol 5: 441-450, 2004.

21. Mailleux AA, Tefft D, Ndiaye D, Itoh N, Thiery JP, Warburton D and Bellusci S: Evidence that SPROUTY2 functions as an inhibitor of mouse embryonic lung growth and morphogenesis. Mech Dev 102: 81-94, 2001.

22. Gross I, Morrison DJ, Hyink DP, Georgas K, English MA, Mericskay M, Hosono S, Sassoon D, Wilson PD, Little M and Licht JD: The receptor tyrosine kinase regulator sprouty1 is a target of the tumor suppressor WT1 and important for kidney development. J Biol Chem 278: 41420-41430, 2003.

23. Basson MA, Akbulut S, Watson-Johnson J, Simon R, Carroll TJ, Shakya R, Gross I, Martin GR, Lufkin T, McMahon AP, et al: Sprouty1 is a critical regulator of GDNF/RET-mediated kidney induction. Dev Cell 8: 229-239, 2005.

24. Klein OD, Minowada G, Peterkova R, Kangas A, Yu BD, Lesot H, Peterka M, Jernvall J and Martin GR: Sprouty genes control diastema tooth development via bidirectional antagonism of epithelial-mesenchymal FGF signaling. Dev Cell 11: 181-190, 2006

25. Minowada G, Jarvis LA, Chi CL, Neubüser A, Sun X, Hacohen N, Krasnow MA and Martin GR: Vertebrate sprouty genes are induced by FGF signaling and can cause chondrodysplasia when overexpressed. Development 126: 4465-4475, 1999.

26. Gross I, Armant O, Benosman S, de Aguilar JL, Freud JN Kedinger M, Licht JD, Gaiddon C and Loeffler JP: Sprouty2 inhibits BDNF-induced signaling and modulates neuronal differentiation and survival. Cell Death Differ 14: 1802-1812, 2007.

27. Yue $J$ and Tigyi G: Conservation of miR-15a/16-1 and miR-15b/16-2 clusters. Mamm Genome 21: 88-94, 2010.

28. Gao Y, Su J, Guo W, Polich ED, Magyar DP, Xing Y, Li H, Smrt RD, Chang Q and Zhao X: Inhibition of miR-15a promotes BDNF expression and rescues dendritic maturation deficits in MeCP2-deficient neurons. Stem Cells 33: 1618-1629, 2015.

29. Hebert SS, Horré K, Nicolaï L, Papadopoulou AS, Mandemakers W, Silahtaroglu AN, Kauppinen S, Delacourte A and De Strooper B: Loss of microRNA cluster miR-29a/b-1 in sporadic Alzheimer's disease correlates with increased BACE1/beta-secretase expression. Proc Natl Acad Sci USA 105: 6415-6420, 2008. 